\title{
Jonas Wolff \\ Del gobierno a la crisis. Acerca del debilitamiento del movimiento indígena ecuatoriano
}

\section{Introducción}

En los años 1990, el movimiento indígena ecuatoriano era considerado como el movimiento étnico más fuerte de América Latina (Yashar 2005: 85; Van Cott 2005: 99). En el marco de un ascenso político espectacular, la Confederación de Nacionalidades Indígenas del Ecuador (CONAIE) y su "brazo político" Pachakutik se convirtieron en desafiantes serios del sistema establecido, de sus representantes y prácticas tradicionales. En enero del año 2000 protagonizaron una rebelión que derrocó al presidente Jamil Mahuad, en el 2003 participaron por unos meses en el gobierno de Lucio Gutiérrez. Desde la ruptura de la alianza con Gutiérrez, sin embargo, el movimiento indígena se encuentra en una situación de crisis. Se ha reducido marcadamente no sólo su rol como protagonista de la protesta social sino también su fuerza política dentro de las instituciones democráticas. El movimiento indígena no desempeñó ningún papel activo ni en la caída del Presidente Gutiérrez en el 2005, ni en la elección de Rafael Correa en 2006, ni durante el primer año del gobierno de Correa en 2007.

En el siguiente artículo se analiza este debilitamiento del movimiento indígena ecuatoriano: Su paso de actor social y político realmente fuerte -un actor capaz de protagonizar la protesta social, capaz de marcar la agenda política, capaz de desafiar a los gobiernos de turno- a actor relativamente marginal con respecto a la política nacional. ${ }^{1}$ Desde la perspectiva del sistema político, este debilitamiento del movimiento indígena significa (por el momento) un "cercamiento" exitoso del desafío indígena. En lo que sigue se atribuye este cercamiento

1 Este artículo se basa en mi análisis del movimiento indígena ecuatoriano en Wolff (2008: Cap. 5). Aquí solo puedo presentar unas líneas muy generales de este análisis. 
político a tres procesos interrelacionados: a la integración política en el sentido de una mayor participación, representación y responsividad; a la cooptación de líderes, grupos y organizaciones (de base) indígenas por instituciones estatales y partidos políticos; y a dinámicas internas -de división y desmovilización- del propio movimiento indígena. Pero antes de analizar estos tres procesos, es preciso resumir brevemente el ascenso político del movimiento indígena ecuatoriano.

\section{El movimiento indígena ecuatoriano}

En los años 1970, se pudo observar una nueva dinámica de movilización y organización indígena tanto en la Amazonía como en la Sierra ecuatoriana. Estos procesos de organización explícitamente indígena confluyeron en 1986 en la creación de la CONAIE. Como confederación nacional, la CONAIE aglutina en su seno a las organizaciones regionales más importantes de los pueblos y nacionalidades indígenas del Ecuador: la Confederación de Nacionalidades Indígenas de la Amazonía Ecuatoriana (CONFENIAE), la Confederación de los Pueblos de Nacionalidad Kichua del Ecuador (ECUARUNARI), y la Confederación de Nacionalidades Indígenas de la Costa Ecuatoriana (CONAICE). ${ }^{2}$

Con el gran "levantamiento indígena" de 1990, la CONAIE apareció en el escenario de la política nacional: Provocado por nuevas concesiones para la explotación de petróleo en territorios indígenas, la CONAIE convocó a protestas que incluyeron el bloqueo de carreteras, marchas masivas, la invasión de latifundios y la toma de edificios públicos (Lucas 2000: 3); en el marco de este levantamiento, la CONAIE puso por primera vez sus reivindicaciones políticas en la agenda nacional (León Trujillo 1994). Le siguieron otros levantamientos indígenas. En 1995, la CONAIE -junto con otros movimientos rurales y urbanos- decidió crear el Movimiento de Unidad Plurinacional Pachakutik - Nuevo País (Beck/Mijeski 1998). A través de Pachakutik se introdujo a las instituciones democráticas: al Congreso Nacional, a

2 Sobre el movimiento indígena ecuatoriano, véanse Barrera Guarderas (2001); Collins (2004); De la Torre (2006); Gerlach (2003); Guerrero (2000); León Trujillo (1994); Lucas (2000); Lucero (2003); Ojeda Segovia (2004); Pallares (2002); Selverston-Scher (2001); Van Cott (2005: Cap. 4); Wolff (2004); Yashar (2005: Cap. 4); Zamosc (2007). 
las diferentes instituciones políticas a nivel subnacional y, en 1997, a la asamblea constituyente de aquél entonces (Andolina 2003). En el marco de la crisis económica en 1999, la CONAIE encabezaba la protesta social contra las medidas de austeridad y ajuste que trataba de imponer el gobierno de Mahuad. Esta escalada de protestas culminó en enero del 2000 en el derrocamiento de Mahuad por una alianza del movimiento indígena con un sector rebelde de las Fuerzas Armadas (Walsh 2001). Durante unas horas, un "triunvirato de salvación nacional" -incluyendo el presidente de la CONAIE, Antonio Vargas- reemplazaba al presidente elegido. El alto comando militar rápidamente terminó esta rebelión que se había convertido en golpe, pero dos años después retornó aquella alianza indígena-militar: En el 2002 ganó las elecciones presidenciales el ex coronel Lucio Gutiérrez con Pachakutik y CONAIE como sus aliados más importantes (Lucas 2003).

A través de sus tres regionales, en los años noventa la CONAIE logró reunir el 80\% de las organizaciones indígenas de base (Van Cott 2005: 107). Por esta razón se considera sin ninguna duda el órgano más representativo de la población indígena que representa entre 30 y 40\% de la población ecuatoriana (Yashar 2005: 2). Sin embargo, la CONAIE no es -y nunca fue- una organización homogénea. Como la población indígena, el movimiento indígena es atravesado por diferentes clivajes: la diversidad étnica entre la población mayoritariamente quichua y las otras "nacionalidades" indígenas que se encuentran especialmente en la Amazonía; ${ }^{3}$ las diferencias religiosas entre católicos y evangélicos; y la división regional entre ECUARUNARI que representa a la mayoría de la población indígena serrana, la importante -pero minoritaria- CONFENIAE y la marginal CONAICE. Además, la CONAIE no es la única confederación indígena. Los competidores nacionales más importantes son la Federación Nacional de Organizaciones Campesinas, Indígenas y Negras (FENOCIN) y la Federación Ecuatoriana de Indígenas Evangélicos (FEINE). La FENOCIN, creada en los años sesenta, es una organización de tipo sindical y tradicionalmente se ubica cerca del Partido Socialista; pero con el ascenso de la CONAIE ha adoptado una gran parte de su discurso étnico. La

3 Oficialmente se distingue la "nacionalidad" quichua (que por su parte consiste en 13 "pueblos" distribuidos por la Sierra y la Amazonía) y 12 otras "nacionalidades" (sobre todo en la Amazonía). 
FEINE fue creada en 1980 como una organización de iglesias evangélicas; en los noventa también aprendió del "modelo éxito" y en 1998 creó incluso su propio partido indígena (Amauta Jatari, luego Amauta Yuyay). Al final de los años noventa, la FEINE -a través de aproximadamente 600 iglesias locales- logró organizar el 17\% de la población indígena (Van Cott 2005: 108-109).

Cuando Lucio Gutiérrez asumió la presidencia, el movimiento indígena llegó por primera vez a formar parte del gobierno nacional. Pachakutik era parte de la coalición gobernante; la líder indígena Nina Pacari ocupó la Cancillería y el ex presidente de la CONAIE, Luis Macas, obtuvo el Ministerio de Agricultura y Ganadería (Barrera et al. 2004). Pero la coalición entre CONAIE/Pachakutik y Gutiérrez no duró más que unos pocos meses. Gutiérrez rápidamente empezó a girar el rumbo hacia alianzas con las élites de la Costa, con los partidos políticos de la derecha, con el gobierno de Estados Unidos y con el Fondo Monetario Internacional (Lucas 2003; Posso 2004). Hasta fines del año 2003, todos sus aliados de campaña declararon la oposición abierta al gobierno de Gutiérrez: no sólo la CONAIE y Pachakutik, sino también otros movimientos sociales urbanos, organizaciones rurales, sindicatos y fuerzas políticas de izquierda en general. Sin embargo, a pesar de las declaraciones de enfrentamiento radical al "traidor" Gutiérrez, este conjunto de organizaciones sociales no fue capaz de movilizar ningún tipo de resistencia significativa al gobierno. Esto no sorprende mucho tratándose de los movimientos sociales urbanos y de los sindicatos ecuatorianos. Pero sí es notable que durante el gobierno de Gutiérrez hayan fracasado todos los intentos por parte de la CONAIE de iniciar un nuevo levantamiento indígena. Las protestas que en el 2005 forzaron la caída de Gutiérrez las protagonizaron justamente los sectores medios de Quito en alianza con los partidos tradicionales. ${ }^{4}$

\section{El "cercamiento" político del movimiento indígena}

¿Cómo se puede explicar este debilitamiento de un actor sociopolítico que hace poco era tan fuerte? Antes que nada, hay que destacar un punto que no tiene relevancia. Es un punto que tiene que ver

4 Sobre la caída de Gutiérrez, véase las contribuciones en Íconos (2005). 
con el hecho de que exista la democracia en el Ecuador. La democracia que realmente existe en este país puede ser muy inestable, bastante formal y distorsionada por poderes y prácticas fácticas, pero aquí muestra su valor: Que el movimiento indígena haya perdido la fuerza social y política que tenía en los años 1990 no resulta de una política de represión. Hubo de vez en cuando acontecimientos aislados de represión, pero en general éstos fracasaron. El ascenso y el éxito, tanto como el debilitamiento y la crisis del movimiento indígena, sólo se pueden entender como dinámicas que se dan en un contexto político que es básicamente democrático.

\subsection{Integración política}

La primera dimensión del argumento que quiero desarrollar, es que la crisis del movimiento indígena es, en parte, el resultado de su propio éxito. Lo conocemos bien de las trayectorias de movimientos sociales en todo el mundo, sobre todo en sistemas políticos democráticos: el ascenso de un nuevo actor colectivo desafiante resulta en una apertura del sistema político. La integración política del nuevo grupo implica su institucionalización, su moderación estratégica y la desmovilización de su base social (Tarrow 1998: 161-175). En este sentido, el empoderamiento de la población indígena causó que el sistema político ecuatoriano se adaptara. La rápida inclusión de Pachakutik en las instituciones democráticas es, por un lado, el resultado de la propia fuerza del movimiento indígena. Pero por otro lado, este éxito se basa en características de la democracia en el Ecuador: El sistema político -precisamente por su fragmentación y el clivaje entre Costa y Sierrafacilita generalmente la incorporación de nuevas fuerzas políticas (León Trujillo 2004). En los años 1990, reformas políticas que profundizaron la descentralización y liberalizaron las leyes electorales y partidarias intensificaron esta permisividad (García Serrano 2005; Van Cott 2005: Cap. 4). El reconocimiento de los pueblos indígenas y de sus propios derechos en la Constitución del 1998 fue un resultado -más bien como un paso más- de este proceso de inclusión. Así Pachakutik rápidamente pasó a ser un partido establecido a nivel nacional con fuerte presencia también a nivel subnacional, sobre todo en la Sierra y la Amazonía. 
Junto a esta integración liberal-democrática, hubo otra forma de integración. Esto se refiere también a los mecanismos de integración formal-institucional, pero de modo colectivo-corporativista. En este sentido, se crearon instituciones estatales que se dedicaron específicamente a reivindicaciones indígenas: la Dirección Nacional de Educación Intercultural Bilingüe (DINEIB), la Dirección Nacional de Salud de los Pueblos Indígenas (DNSPI) y el Consejo de Desarrollo de las Nacionalidades y Pueblos del Ecuador (CODENPE). En todas estas instituciones las propias organizaciones indígenas lograron extensas posibilidades de participación, administración y hasta autogestión (García Serrano 2003: 212; 2005: 4; Almeida/Arrobo Rodas 2004: 54-62). Además, hay una dimensión informal de inclusión política: Con sus levantamientos, la CONAIE logró establecerse como la representante públicamente reconocida de los intereses y valores de la población indígena del país. Si decisiones o medidas políticas amenazaban intereses vitales de la población indígena, la CONAIE podía convocar a protestas para obligar al presidente de turno a negociar con ella. Esta práctica informal de lograr respuestas políticas a demandas indígenas no sólo se refiere a la confederación nacional: Habiendo establecido su fuerza movilizadora, la capacidad por parte de las organizaciones indígenas de todos los niveles de negociar exitosamente con "el Estado" era mucho mayor que antes.

Lo dicho hasta ahora suena como una historia de éxito total. Pero es una historia muy parcial. Sin lugar a duda, es impresionante el aumento de reconocimiento, de participación y la representación política que ha conseguido el movimiento indígena en tan corto tiempo y con estrategias tan pacíficas. El problema es que este éxito no ha logrado convertirse en un mejoramiento de la situación socio-económica de la población indígena. Así, un informe del Banco Mundial (Hall/Patrinos 2005) muestra que las tasas de pobreza indígena no han disminuido entre 1994 y 2003. Las concesiones materiales que la CONAIE y sus aliados consiguieron en el transcurso de las diferentes protestas o quedaron en el papel sin mayores consecuencias o fueron medidas defensivas y de corto plazo o produjeron un alivio limitado a comunidades específicas (García Serrano 2003: 214; Wolff 2008: 269-272). Sin embargo, también hubo avances importantes respecto a cuestiones particulares, pero eso no pone en duda el punto general: No se puede entender el debilitamiento del movimiento indígena como una reac- 
ción de la población indígena al hecho de que ya no haya más reivindicaciones urgentes.

\subsection{Cooptación política y división interna}

Obviamente el debilitamiento del movimiento indígena no es el simple resultado de un "ciclo de contención" (Tarrow 1998) que haya resultado en la integración política exitosa del actor social y sus reivindicaciones. Tales procesos de integración son reales. Sin embargo, como se argumenta en los siguientes párrafos, hay dos procesos complementarios que también juegan un rol importante: la cooptación de líderes, grupos y organizaciones indígenas por instituciones estatales y partidos políticos; y las dinámicas de división y desmovilización internas al movimiento indígena.

Los mencionados procesos de integración parcial desde el inicio causaron tensiones dentro del movimiento indígena. Con la entrada al juego político surgieron clivajes entre dirección y base dentro de cada una de las organizaciones y se agravaron los conflictos de competencia entre las diferentes organizaciones. Al mismo tiempo, la apertura política fue acompañada por estrategias estatales que apuntaban a profundizar estas tensiones a través de la cooptación focalizada de líderes y segmentos del movimiento indígena. Durante el gobierno de Lucio Gutiérrez, ambos procesos se intensificaron: La decepción por el fracaso en la participación del gobierno potenció las dinámicas internas de debilitamiento y división. Y el presidente las empujó con más recursos y más habilidades que sus predecesores (Beck/Mijeski 2006; Wolff 2007).

Con respecto a las dinámicas internas se pueden distinguir tres dimensiones:

- la tensión programática entre un proyecto de cambio socio-político de largo plazo y una agenda de reivindicaciones urgentes;

- el llamado "efecto Pachakutik" (García Serrano 2005: 8) que tiene que ver con las conocidas repercusiones que tiene la participación política en un movimiento social; y

- la competencia estructural dentro del movimiento indígena.

En cada una de estas tres dimensiones se puede analizar cómo los gobiernos de turno conectan sus estrategias a las dinámicas internas 
del movimiento indígena para cooptarlo, dividirlo y marginarlo como actor político.

Luego del derrocamiento de Mahuad en enero del 2000 se agravó la tensión programática. El nuevo presidente Gustavo Noboa siguió aplicando por un lado la misma agenda macropolítica de Mahuad: implementó la dolarización de la economía, llevó adelante una serie de reformas de ajuste y estabilización macroeconómica y trató de consolidar una alianza partidaria de centro-derecha (Wolff 2003: Cap. 4). Pero por otro lado, se abrió al diálogo con organizaciones y representantes indígenas y estaba dispuesto a satisfacer ciertas demandas concretas. Entonces, la CONAIE entró en negociaciones e incluso después del fracaso del diálogo, los alcaldes y prefectos de Pachakutik siguieron negociando acerca de la distribución de recursos financieros y proyectos de desarrollo (Collins 2004: 54). Aquí es importante destacar que estos problemas no (o no sólo) demuestran una falta de capacidad de pensamiento y consistencia estratégica, sino que reflejan un dilema estructural: Dentro del movimiento indígena hay, con toda la razón, el interés de tener un impacto en las líneas generales de la política nacional y, al mismo tiempo, existe una presión por parte de la base social de conseguir rendimientos palpables.

El dilema de perseguir una agenda de cambio macropolítico y al mismo tiempo realizar logros concretos -más bien micropolíticosafectó especialmente a los representantes de Pachakutik en el ámbito nacional. Como fracción minoritaria en el parlamento nunca fueron capaces de lanzar proyectos relevantes de reforma; en un Congreso altamente fragmentado y caracterizado por prácticas oportunistas y clientelistas prácticamente no quedaba otra alternativa más que "lanzarse al juego": negociar con las diferentes fuerzas políticas y tratar de llegar a acuerdos concretos. ${ }^{5}$ Pero era bien claro que así no se llegaría a iniciar un proceso de cambio político ni a lograr beneficios palpables para las comunidades indígenas. En consecuencia, desde la CONAIE y la población indígena en general creció la crítica hacia "su brazo político” (Beck/Mijeski 2006; Collins 2004; García Serrano 2005).

5 Pero hay que decir que Pachakutik también se mostró altamente vulnerable frente a las prácticas políticas dominantes en el Congreso. Por ejemplo, después de la ruptura con el presidente Gutiérrez, cinco de los once diputados de Pachakutik decidieron apoyar casi abiertamente al gobierno (ElUniverso.com, 10/1/2005; cf. Beck/Mijeski 2006). 
Al mismo tiempo, la pérdida de crédito de Pachakutik en la base indígena afectó también a la conducción de la CONAIE. En un sentido de "inclusión corporativa" (De la Torre 2006: 254), la CONAIE en la política nacional tenía que presentarse como un actor unitario capaz de participar en los debates nacionales y en las negociaciones políticas de la élite. Esta necesidad contradijo a la vez los principios de representación plural, de conexión fuerte entre dirección y base y de control social de la dirigencia que, en general, caracterizan al movimiento indígena (Ströbele-Gregor 2008: 137-138). Con sus ofertas clientelistas, Gutiérrez se aprovechó de este distanciamiento entre dirección y base y lo profundizó aún más: A través del aparato estatal, especialmente a través de diversos programas sociales e inversiones en proyectos de infraestructura local, pero también a través de su partido Sociedad Patriótica, Gutiérrez consolidó redes clientelares a nivel local para "disputarle al movimiento indígena su centro fundamental: las comunidades" (Dávalos 2005: 62).

Pero la estrategia de Gutiérrez de atar la población indígena directamente a sí mismo, a su gobierno y a su partido, no hubiera sido capaz de minar un movimiento tan fuerte, un movimiento tan anclado en las comunidades indígenas. Aquí entra como factor importante la fragmentación del propio movimiento indígena. La competencia estructural entre las diversas organizaciones indígenas (Bretón Solo de Zaldívar 2001: 313, 181-182; Lucero 2006) hizo posible el divide et impera forzado por Gutiérrez. De esta manera, Gutiérrez no tuvo que romper los lazos con el movimiento indígena, sino que sencillamente cambió de aliados indígenas. Después de la ruptura con Pachakutik y CONAIE, el presidente intensificó las ofertas hacia la FEINE, la FENOCIN y las regionales de la CONAIE en la Amazonía y la Costa. A ellas, les ofreció proyectos de desarrollo, inversiones en infraestructura y regalos estatales ("picos y palas"). Incorporó así mismo representantes de la FEINE y la CONFENIAE al gobierno. Un ejemplo es el nombramiento de Antonio Vargas como Ministro de Bienestar Social. Vargas es un líder histórico del movimiento indígena de la Amazonía, era presidente de la CONAIE durante la rebelión contra Mahuad, y se postuló como candidato presidencial de la FEINE en las elecciones del 2002. Vargas tenía por lo tanto relaciones importantes tanto con la parte amazónica de la CONAIE como con el rival más importante de la CONAIE. Otro mecanismo para disputarle a la CO- 
NAIE las comunidades indígenas aprovechándose de la fragmentación competitiva del movimiento indígena era la cooperación por parte de Gutiérrez con un grupo de líderes indígenas disidentes de la CONAIE: la Federación de Pueblos Indígenas, Campesinos y Negros del Ecuador (FEDEPICNE): Desde el inicio de su presidencia, Gutiérrez incorporó representantes de esta organización (hasta entonces virtual) a su gobierno y utilizó FEDEPICNE como canal para distribuir recursos y cargos estatales. Gutiérrez también usó las instituciones indígenasestatales para minar la posición hegemónica de la CONAIE dentro del movimiento indígena: En la dirección del CODENPE -la institución estatal que implementa proyectos de desarrollo en y con las comunidades indígenas- Gutiérrez cambió el esquema de representación por nacionalidades y pueblos (que favorecía a la CONAIE) por un esquema de representación corporativa que incluyó a la FEINE y a la FENOCIN. Así, Gutiérrez logró cooptar la FEINE y una parte importante de la CONFENIAE. Lo último efectivamente dividió a la CONAIE en la Amazonía. Pero hasta la corriente opositora de la CONFENIAE expresó cierta cercanía al gobierno y decidió cooperar pragmáticamente con Gutiérrez. Por ejemplo, un dirigente de la CONFENIAE, en una entrevista en enero del 2005, destacó que

la región amazónica nunca ha tenido tal oportunidades [...]. Esta vez, con el apoyo del ministro Antonio Vargas, con el apoyo del gobierno, por la coyuntura política que hay, estamos llegando con proyectos a las nacionalidades, a las comunidades, que sí necesitan proyectos de desarrollo. ${ }^{6}$

El resultado fue inequívoco: Cuando la dirigencia serrana de la CONAIE intentaba convocar a protestas, a un nuevo levantamiento contra el gobierno de Gutiérrez, nunca recibía apoyo suficiente: ni de sus rivales FEINE o FENOCIN, ni de sus regionales de la Amazonía o la Costa, ni de su propia base en la Sierra que no veía al gobierno de Gutiérrez como una amenaza.

\section{Conclusión}

La primera conclusión que resulta de este análisis del debilitamiento del movimiento indígena en el Ecuador es que la democracia ecuatoriana, realmente existente -con toda su inestabilidad estructural-, tiene

6 Entrevista del autor con Eduardo Mamallacta, Dirigente de Territorio, CONFENIAE, 28 de enero de 2005, Quito. 
una capacidad impresionante de adaptarse a desafíos sociopolíticos "desde abajo". En segundo lugar, para entender el manejo político de conflictos sociales en la región andina, es importante tener en cuenta la cultura política caracterizada por el clientelismo, el corporativismo y por procesos informales y particulares de negociación. Tercero, los procesos de movilización y politización indígena contienen características ambivalentes: Marcado por el objetivo de contribuir a una alternativa democrática-participativa a la política tradicional, el movimiento indígena se mostró altamente vulnerable frente a la cultura política dominante $\mathrm{o}$, mejor dicho, a las prácticas políticas dominantes.

En este sentido se puede entender la doble reacción del movimiento indígena a su crisis, una reacción que consiste básicamente en la combinación de una agenda de rechazo con una agenda específicamente indígena. Por un lado, se enfatiza todo lo que tiene que ver con la defensa de intereses vitales. Esto se mostró durante el levantamiento del 2006 contra el Tratado de Libre Comercio con los Estados Unidos y contra la petrolera Occidental. De esta manera, la dirigente indígena serrana, Blanca Chancoso, resume la agenda macropolítica de la CONAIE como una agenda de rechazo: "No al Plan Colombia, no al TLC, no a la base militar en Manta, no al pago de la deuda externa" (Fuentes 2007). Por otro lado, la estrategia consiste en lo que el ex presidente de la CONAIE, Luis Macas, ha llamado la necesidad de "volver a lo nuestro" (Macas 2005a). La idea detrás de este eslogan es reunir el movimiento indígena a través de cierta retirada del juego macropolítico y de un proyecto que era más político que indígena. Como lo dijo Macas:

volver a los temas eje, que fueron articuladores del movimiento indígena, como el territorio y la educación y dejar los temas que han causado la dispersión, como la participación en temas políticos (Macas 2005b).

Por consiguiente, alrededor de las elecciones del 2006 y 2007 se pudo observar una "indigenización" de CONAIE y Pachakutik, una indigenización no sólo en términos programáticos, sino también en términos estratégicos: Pachakutik se presentó más claro que nunca como un partido meramente indígena; en consecuencia, grupos urbano-mestizos que antes constituían aliados importantes para este movimiento político salieron del partido (Beck/Mijeski 2006: 17). Esta tendencia se ha reforzado durante el actual gobierno de Rafael Correa. Desde su 
elección a finales del 2006, Correa posee la hegemonía política en todo lo que tiene que ver con un cambio social y político que enfrenta a los adversarios tradicionales del movimiento indígena: al "neoliberalismo" y a la "partidocracia". Lo que deja Correa para el movimiento indígena es la agenda específicamente indígena, todas las demandas relacionadas con la idea del Ecuador como un Estado "plurinacional". ${ }^{7}$

Dadas las experiencias del movimiento indígena dentro del juego político "à la ecuatoriana", se puede entender muy bien esta tendencia por parte del movimiento indígena a dedicarse a una agenda que se concentre en defender y perseguir ciertos intereses comunes, pero particulares de la población indígena. Pero si es que tratamos de pensar en una profundización de la democracia -como la que promete el gobierno de Rafael Correa-, es preciso que no se regrese a la "representación ventrílocua" (Guerrero 1997) del pasado: No basta que un gobierno como el de Correa posiblemente considere a la población indígena, sus intereses y valores, sino que es importante que las propias organizaciones indígenas participen activamente en los debates nacionales y en todas las decisiones macropolíticas. Que se escuche a representantes indígenas cuando se trata de cuestiones como la educación bilingüe es pertinente y obviamente importante, pero el logro del movimiento indígena ecuatoriano de las últimas décadas ha sido que las voces indígenas hayan empezado a tener peso en todos los temas de la política nacional, sea la política económica, social o exterior.

7 Por consiguiente, en el ámbito de la reciente Asamblea Constituyente los éxitos más importantes de la CONAIE fueron la declaración del Ecuador como Estado "plurinacional" y la inclusión de los idiomas quichua y shuar como idiomas oficiales (“de relación intercultural”) en la nueva constitución (cf. Ecuador 2008). 


\section{Bibliografía}

Almeida, Ileana/Arrobo Rodas, Nidia (2004): “Autonomía Indígena Frente Al Estado". En: <www.latautonomy.org/ECUADORLIBRO_LATAUTONOMY.pdf> (16.06.2005).

Andolina, Robert (2003): "The Sovereign and its Shadow: Constituent Assembly and Indigenous Movement in Ecuador”. En: Journal of Latin American Studies, 35, 4, pp. 721-750.

Barrera, Augusto (2001): Acción Colectiva y Crisis Política. El Movimiento Indígena Ecuatoriano en la Década de los Noventa. Quito: Abya-Yala.

Barrera, Augusto et al. (2004): Entre la utopía y el desencanto. Pachakutik en el gobierno de Gutiérrez. Quito: Editorial Planeta.

Beck, Scott H./Mijeski, Kenneth J. (1998): "Mainstreaming the Indigenous Movement in Ecuador: The Electoral Strategy" (Ponencia en el XXI International Congress of the Latin American Studies Association, 24-26.09.1998, Chicago, IL). En: <www.168.96.200.17/libros/lasa98/Mijeski-Beck.pdf> (21.01.2005).

- (2006): "How to Lose by Winning: The Ecuadorian Indigenous Movement After the 2002 Elections" (Ponencia en el XXVI International Congress of the Latin American Studies Association, 15-18.03.2006, San Juan, PR).

Bretón Solo de Zaldívar, Víctor (2001): Cooperación al desarrollo y demandas étnicas en los Andes ecuatorianos. Quito: FLACSO.

Collins, Jennifer (2004): "Linking Movements and Electoral Politics. Ecuador's Indigenous Movement and the Rise of Pachakutik". En: Burt, Jo-Marie/Mauceri, Philip (eds.): Politics in the Andes. Identity, Conflict, Reform. Pittsburgh: University of Pittsburgh Press, pp. 38-57.

Dávalos, Pablo (2005): "Ecuador: Plan Colombia, crisis institucional y movimientos sociales”. En: Revista OSAL (Observatorio Social de América Latina), 6, 18, pp. 57-67.

De la Torre, Carlos (2006): "Ethnic Movements and Citizenship in Ecuador". En: Latin American Research Review, 41, 2, pp. 247-259.

Ecuador 2008: Constitución del Ecuador. En: <www.asambleanacional.gov.ec/ images/constitucion_de_bolsillo.pdf $>$ (01.12.2008).

Fuentes, Federico (2007): "Ecuador: The Indigenous Movement and Correa". En: International News/Green Left Weekly, 720 (<www.greenleft.org.au>; 13.02. 2008).

García Serrano, Fernando (2003): "Política, estado y diversidad cultural: A propósito del movimiento indígena ecuatoriano". En: Bretón, Víctor/García, Francisco (eds.): Estado, etnicidad y movimientos sociales en América Latina. Ecuador en crisis. Barcelona: Icaria, pp. 193-215.

- (2005): "De movimiento social a partido político: el caso del movimiento de unidad plurinacional Pachakutik-Ecuador" (Ponencia en el Seminario Internacional del Instituto Rosa Luxemburg Stiftung, 30.10.-01.11. 2005, São Paulo). En: $<$ www.rls.org.br/publique/media/PartAL_Garcia.pdf> (15.12.2006).

Gerlach, Allen (2003): Indians, Oil, and Politics. A Recent History of Ecuador. Wilmington: Scholarly Resources. 
Guerrero, Andrés (1997): "Poblaciones indígenas, ciudadanía y representación”. En: Nueva Sociedad, 150, pp. 98-105.

- (ed.) (2000): Etnicidades. Quito: FLACSO Ecuador.

Hall, Gillette/Patrinos, Harry Anthony (2005): "Indigenous Peoples, Poverty and Human Development in Latin America: 1994-2004. Executive Summary”. En: $<$ www.worldbank.org> (19.05.2005).

Íconos (2005): “Dossier: La caída de Gutiérrez y la rebelión de abril”. En: Íconos, 23, pp. 19-110.

León Trujillo, Jorge (1994): De campesinos a ciudadanos diferentes. El levantamiento indigena. Quito: Abya-Yala.

- (2004): "La democracia real versus la democracia idealizada. Ecuador de 1978 a 2003”. En: Revista Política, 42, pp. 87-128.

Lucas, Kintto (2000): We will not Dance on our Grandparents' Tombs: Indigenous Uprisings in Ecuador. London: Catholic Institute for International Relations.

- (2003): El movimiento indigena y las acrobacias del Coronel. Quito: Tinají.

Lucero, José Antonio (2003): "Locating the 'Indian Problem': Community, Nationality and Contradiction in Ecuadorian Indigenous Politics". En: Latin American Perspectives, 30, 1, pp. 23-48.

- (2006): "Representing 'Real Indians'. The Challenges of Indigenous Authenticity and Strategic Constructivism in Ecuador and Bolivia". En: Latin American Research Review, 41, 2, pp. 31-56.

Macas, Luis (2005a): "Volver a lo nuestro". Entrevista en: BBC Mundo, 16.09.2005 (<www.bbcmundo.com>; 01.09.2006).

- (2005b): "Recuperar el rol social de CONAIE". Entrevista con Elisa Maturana en Oxfam America, 07.07.2005 (<www.oxfamamerica.org>; 18.02.2008).

Ojeda Segovia, Lautaro (2004): "Análisis politológico del procedo de construcción de la autonomía multicultural en el Ecuador". En: <www.latautonomy.org/ EstudioPolitico_Ecuador.pdf $>$ (20.08.2004).

Pallares, Amalia (2002): From Peasant Struggles to Indian Resistance. The Ecuadorian Andes in the Late Twentieth Century. Norman: University of Oklahoma.

Posso, Antonio (2004): Radiografía de una traición. Quito: Editorial El Conejo.

Selverston-Scher, Melina (2001): Ethnopolitics in Ecuador. Indigenous Rights and the Strengthening of Democracy. Coral Gables: North-South Center Press.

Ströbele-Gregor, Juliana (2008): "Indigene Völker in der politischen Arena: Zum Verständnis von Politik und Demokratie bei indigenen Völkern in der Andenregion“. En: Lateinamerika Analysen, 7, 2, pp. 125-140.

Tarrow, Sidney (1998): Power in Movement: Social Movements and Contentious Politics. New York: Cambridge University Press.

Van Cott, Donna Lee (2005): From Movements to Parties in Latin America. The Evolution of Ethnic Politics. Cambridge: Cambridge University Press.

Walsh, Catherine E. (2001): "The Ecuadorian Political Irruption. Uprisings, Coups, Rebellions, and Democracy”. En: Nepantla: Views from South, 2, 1, pp. 173-204. 
Wolff, Jonas (2003): Bestimmungsfaktoren und Konsequenzen der offiziellen Dollarisierung in Lateinamerika. Eine politökonomische Analyse unter besonderer Berücksichtigung Ecuadors. Hamburg: Institut für Iberoamerika-Kunde (Beiträge zur Lateinamerikaforschung, Band 10).

- (2004): Demokratisierung als Risiko der Demokratie? Die Krise der Politik in Bolivien und Ecuador und die Rolle der indigenen Bewegungen. Frankfurt am Main: Hessische Stiftung Friedens- und Konfliktforschung (HSFK-Report 6/2004).

- (2007): “(De-)Mobilising the Marginalised. A Comparison of the Argentine Piqueteros and Ecuador's Indigenous Movement". En: Journal of Latin American Studies, 39, 1, pp. 1-29.

- (2008): Turbulente Stabilität. Die Demokratie in Südamerika diesseits ferner Ideale. Baden-Baden: Nomos (Studien der Hessischen Stiftung Friedens- und Konfliktforschung, Band 1).

Yashar, Deborah J. (2005): Contesting Citizenship in Latin America. The Rise of Indigenous Movements and the Postliberal Challenge. Cambridge: Cambridge University Press.

Zamosc, León (2007): "The Indian Movement and Political Democracy in Ecuador". En: Latin American Politics \& Society, 49, 3, pp. 1-34. 
